\title{
Search for spectroscopical signatures of transiting HD 209458b's exosphere*
}

\author{
C. Moutou ${ }^{1}$, A. Coustenis ${ }^{2}$, J. Schneider ${ }^{2}$, R. St Gilles ${ }^{2}$, M. Mayor ${ }^{3}$, D. Queloz ${ }^{3}$, and A. Kaufer ${ }^{1}$ \\ 1 ESO, Alonso de Cordova 3107, Santiago de Chile \\ e-mail: akaufer@eso.org \\ 2 Observatoire de Paris Meudon, 92195 Meudon Cedex, France \\ e-mail: Athena.Coustenis@obspm.fr, Jean.Schneider@obspm.fr, Richard.Saint-Gilles@obspm.fr \\ 3 Observatoire de Genève, Switzerland \\ e-mail: michel.mayor@obs.unige.ch, didier.queloz@obs.unige.ch
}

Received 13 November 2000 / Accepted 18 January 2001

\begin{abstract}
Following recent attempts to detect the exosphere of the extra-solar planet 51 Pegb in the infrared (Coustenis et al. 1997, 1998; Rauer et al. 2000a), we discuss here a search for optical spectroscopic signatures from a gaseous extended envelope (called exosphere) surrounding the planet HD 209458b. This planet has a demonstrated photometric transit (Charbonneau et al. 2000a; Henry et al. 2000), thus offering an increased probability for the spectroscopic detection of such an envelope. Therefore it is the best known candidate for probing the exospheric composition of a giant planet, orbiting a Sun-like star at a short distance. The observations were performed with UVES at the VLT and cover most of the 328-669 nm range. We did not detect HD 209458b's exosphere at a level of $1 \%$, a value close to the predictions. We discuss here the first results obtained and their limitations, as well as future prospective.
\end{abstract}

Key words. techniques: spectroscopic - stars: atmosphere - stars: planetary systems

\section{Introduction}

The research on extra-solar planets has grown considerably with radial velocity surveys (Mayor \& Queloz 1995; Marcy \& Butler 1996; Hatzes et al. 1996) in the past 5 years. Almost 50 such systems have been discovered to date (September 2000), all with the radial-velocity technique. Most of these new systems are giant planets (Jupiter-like) with extremely short periods, i.e., the ones which show the largest observed Doppler shift in the stellar reflex motion. This kind of system was not expected from theories of planetary system formation, until models of giant planets migrating through the young dust disk were developed (Lin et al. 1996). With the radial velocity techniques, however, the orbit inclination is not known, which leads to an undetermined planet mass $M \sin i$.

Looking at the models describing a giant gaseous planet that has migrated to a short distance from its star, we may expect that an extended envelope of gas ("exosphere") is formed. Spectral signatures of this exosphere could be present in the transmission spectrum of the system, i.e., during a transit (Schneider et al. 1998). Such

Send offprint requests to: C. Moutou, e-mail: cmoutou@eso.org

* Based on public data from the UVES Commissioning at the ESO 8.2 m Kueyen telescope operated on Paranal Observatory, Chile. searches have already been conducted in the infrared, in the ground- and space-based spectra of 51 Peg's system (Coustenis et al. 1997; Coustenis et al. 1998; Rauer et al. 2000a), so far without a clear indication of a detection.

The star HD 209458 shows a Doppler shift amplitude of $86 \mathrm{~m} \mathrm{~s}^{-1}$, and a period of 3.52433 days (equal to a separation of $0.045 \mathrm{AU}$ ) (Mazeh et al. 2000; Henry et al. 2000). Follow-up observations in photometry have shown that the star is eclipsed by the close-by planet (Charbonneau et al. 2000a; Henry et al. 2000). The geometry of the system is therefore set. Namely, from the duration and the depth of the planetary transit we derive the planet radius and the orbit inclination. Finally, with the two techniques we measure the following characteristics: HD 209458b has 0.69 Jupiter mass and 1.4 Jupiter radius. For the first time, it was thus possible to prove the planetary nature of the orbiting companion, and to assess that the invisible planet is gaseous. Recently, higher accuracy photometry with HST allowed one to probe if the planet has rings or satellites (Brown et al. 2001), while from an analysis of HD 209458b's spectroscopic transit, the orientation of the planet's rotational axis was derived (Queloz et al. 2000).

This paper presents our first attempt in detecting spectroscopic signatures of the exosphere of HD 209458b, for which the geometry is favorable, by using UVES, the UV-Visual Echelle Spectrograph mounted on the VLT 


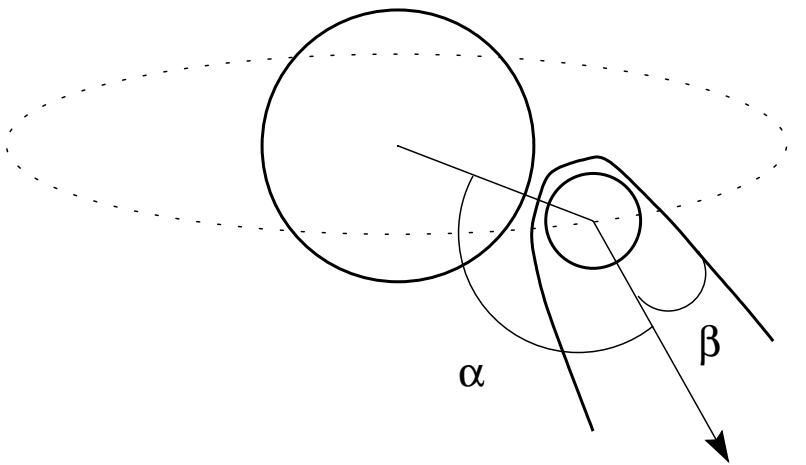

Fig. 1. Illustration of a cometary-tail exosphere around HD 209458b. Stellar wind, escape velocities of the particles and tangential movement of the planet on its orbit define the angles $\alpha$ and $\beta$ (see Sect. 2)

telescope "Kueyen". Implications, perspective and comparison with similar observations are discussed.

\section{Transmission spectrum}

When considering the forces exerted on the external atmosphere of a close-by, giant planet - gravity of the planet, radiation pressure and stellar wind from the parent star - it is predicted that chemical species efficiently escape the planet's atmosphere, forming an extended and tenuous envelope (Chassefière 1996), while the planet body remains stable amongst evaporation (Guillot et al. 1996). From the total UV luminosity of a G2 star, the temperature and radius of the exosphere are expected to be, respectively, $10^{4} \mathrm{~K}$ and $10 R_{\text {Jup }}$, i.e. the radius is larger than the Roche radius (given by $a\left(M_{\mathrm{pl}} / 3 M_{*}\right)^{1 / 3}=4 R_{\mathrm{Jup}}$ ) (Coustenis et al. 1998).

We expect a mass transfer from the planet to a circumstellar accretion ring with a critical radius: $a_{\mathrm{c}}=a(1+$ $q)(b / a)^{4}$, where $b$ is the distance of the Lagrange point L1 to the star and $q$ the mass ratio $M_{\mathrm{pl}} / M_{*}$ (Schneider et al. 1998). Since $q$ is close to 0 , one finally has $a_{\mathrm{c}} \simeq a$ : the evaporated matter remains on the planet orbit. But we must in addition take into account a possible stellar wind which may transform the ring into a comet-like tail of the giant planet similar to the Venus tail observed in situ (e.g. Brace et al. 1987). The tail is characterized by two parameters (Fig. 1):

- Its opening angle $\beta$ given by $\tan \beta=V_{\text {esc }} / V_{w}$, where $V_{\text {esc }}$ is the velocity of evaporated ions when they escape the planet and $V_{w}$ is the radial velocity of ions due to the stellar wind and is approximately equal to the wind velocity;

- The angle $\alpha$ between the tail and the star-planet radial direction, due to the planet's orbital revolution: $\tan \alpha=V_{\mathrm{pl}} / V_{w}$, where $V_{\mathrm{pl}}$ is the tangential velocity of the planet.

The species present in the tail are highly heated, ionized and have large escape velocities (Chassefière 1997). The resulting absorption lines could thus appear blueshifted and present some broadening. In the atmospheres of extrasolar giant planets located at short distance from their parent star, $\mathrm{CO}$ is believed to be more abundant than $\mathrm{CH}_{4}$ due to high temperatures, and clouds are more likely to be formed from silicates - rather than ammonia as on Jupiter - (Guillot et al. 1996; Goukenleuque et al. 2000). The dominant expected ionized species in the extended tail are $\mathrm{CH}^{+}, \mathrm{CO}^{+}, \mathrm{N}_{2}^{+}$and $\mathrm{H}_{2} \mathrm{O}^{+}$(Coustenis et al. 2000), which all possess observable transition lines in the optical. The effective absorption of these lines, spread over the optical range, is expected to be of the order of 1 to $2 \%$ (Coustenis et al. 1998). This surprisingly high value comes from the large extension of the tail.

Alternative scenarios of the envelope geometry could be encountered, like a torus of matter - if the planet has a strong magnetic field - or infalling bridges of matter from the planet to the star, while the comet-like tail seems to be the most likely scheme.

The simulated atomic transmission spectrum of the exosphere shows the predominance of alkali metal lines (Seager \& Sasselov 2000). The strongest absorption lines of the planet exosphere atomic content are expected to originate from NaI, HI and HeI. Note however that this model does not take into account the complex photochemistry - involving the molecular ions mentioned above - taking place in the exosphere.

\section{Observations}

Observations of HD 209458 were performed with UVES (D'Odorico et al. 2000) during its second commissioning run in December 1999. We observed the transit during only $37 \mathrm{~min}$ (of the 2.5 hours of transit duration) on Dec. 17th, due to the star's low position on the sky. In addition, $29 \mathrm{~min}$ were allocated to observe the star one night later, out of transit. The total integration time was separated into three exposures, in order not to saturate the detectors, to distinguish between noise and real spectral features, and to eventually obtain some time information (Table 1). The data is released for public use (see http://www .eso.org/science/uves_comm/).

The achieved spectral resolution is 58000 in the blue arm and 71000 in the red arm of UVES, with 0.6 and $0.7^{\prime \prime}$ respective slit widths. The spectral domain covered with the Dichroic- $1-390+564$ setting is: $328-456.2 \mathrm{~nm}$ (blue arm, "B"), 458.3-564.4 nm (red arm, EEV detector, "RU") and 565.4-668.6 nm (red arm, MIT detector, "RL"). Table 1 also gives indications on the observing conditions.

A comparison star of early spectral type was also observed at similar airmass, for the removal of telluric features, whereas the best comparison spectrum for recognizing and eliminating the stellar absorption features is HD 209458 itself, observed one night later. It was recognized only afterwards, during the data analysis, that a further comparison star of similar spectral type as HD 209458 would have been another valuable reference for a cross 
Table 1. Dates and conditions of observations of the star HD 209458 during both the transit night ("ON") and the following night ("OFF"), and the corresponding phase of the planet's orbit. The transit time was Dec. 17th at 00:23:42 hour (Julian date 2451529.51646, orbital phase $=0$ ), predicted from ephemeris of IAU circular 7315 (D. Charbonneau et al. 1999). The observing conditions as well as the SNR in each part of the spectrum is also given. It was measured on small continuum sections

\begin{tabular}{|l|c|c|c|c|c|c|}
\hline Exposure & $\begin{array}{c}\text { Julian date } \\
\text { of observation }\end{array}$ & $\begin{array}{c}\text { orbital } \\
\text { phase }\end{array}$ & $\begin{array}{c}\text { integration } \\
\text { time (sec) }\end{array}$ & $\begin{array}{c}\text { SNR } \\
(\mathrm{B} / \mathrm{RL} / \mathrm{RU})\end{array}$ & Airmass & $\begin{array}{c}\text { Seeing at } \\
\text { airmass } 1\left(^{\prime \prime}\right)\end{array}$ \\
\hline HD 209458-ON 1 & 2451529.506077 & 0.00054 & 600 & $110 / 170 / 270$ & 2.16 & 0.90 \\
HD 209458-ON 2 & 2451529.513557 & 0.00264 & 900 & $140 / 240 / 340$ & 2.31 & 0.90 \\
HD 209458-ON 3 & 2451529.525691 & 0.00610 & 700 & $130 / 220 / 340$ & 2.61 & 0.80 \\
\hline HD 209458-OFF 1 & 2451530.5093424 & 0.28520 & 600 & $140 / 230 / 360$ & 2.27 & 0.55 \\
HD 209458-OFF 2 & 2451530.5168301 & 0.28732 & 540 & $130 / 220 / 310$ & 2.45 & 0.60 \\
HD 209458-OFF 3 & 2451530.5236172 & 0.28925 & 600 & $120 / 210 / 340$ & 2.64 & 0.58 \\
\hline
\end{tabular}

Table 2. Description of the observed stars

\begin{tabular}{|l|c|c|c|c|c|}
\hline Star & $V$ & Sp Type & RA(2000) & DEC(2000) & Comment \\
\hline HD 209458 & 7.65 & G0V & $22: 03: 11$ & $+18: 53: 04$ & main target star \\
HD 216494 & 5.78 & B8IV/V & $22: 53: 28.7$ & $-11: 36: 59$ & ref. observed after the On-transit \\
HD 224926 & 5.11 & B7III-IV & $00: 01: 50$ & $-03: 01: 39$ & ref. observed after the Off-transit \\
\hline
\end{tabular}

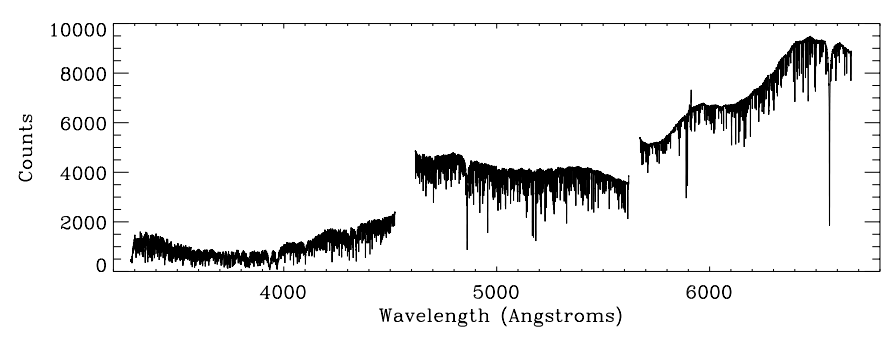

Fig. 2. The total spectrum of the star HD 209458 obtained with UVES, after flatfielding, optimal extraction and spectral rebinning. Some gaps can be seen, which are intrinsic to observations in the DIC1 $-390+564 \mathrm{~nm}$ setting. The total observed range is $3280-4562 \AA, 4583-5644 \AA$ and $5654-6686 \AA$. Resolving powers of 58000 in the blue and 71000 in the red are achieved

check of stellar contribution. Table 2 gives some parameters of the observed stars.

The data reduction was done by using the UVES context of the ESO-MIDAS reduction package as it is used in the VLT data reduction pipeline. The use of this context allows one to benefit from the MIDAS scripts with a step-by-step control of the data processing, thus allowing a deeper understanding of the noise residuals. For instance, we were able to check the spectra directly after extraction, previous to any spectral rebinning. The optimal extraction method with cosmic removal was used. The calibration data (arc and flatfield lamp) were obtained directly after the target exposures, as the instrument stability was not yet fully established.

Figure 2 shows the total spectrum obtained during the transit of HD 209458, after flatfielding, optimal extraction and spectral rebinning; no response correction was performed. The displayed spectrum is the average of all three exposures.

\section{Analysis, limitations and conclusions}

\subsection{Achieved signal-to-noise ratio}

The noise was measured over each section of the spectrum and the resulting averaged SNR is given in Table 1. Small sections without stellar lines were selected for this estimate.

Summing up the three exposures, the total SNR per spectral range (Blue/Red-low/Red-up) in the continuum is: $200 / 300 / 500$ during the on-transit night, and similar values for the off-transit set of observations. In the stellar lines, the SNR can be up to one half of the value.

Previous to any line search, the spectra were empirically shifted to the solar spectrum, taken as a velocity reference frame (Delbouille et al. 1973). It is also necessary to shift the OFF spectrum versus the ON in velocity, in order to adjust the relative position of stellar lines. This was done by shifting the starting wavelength of one of the spectra and rebinning the spectra at a higher sampling by interpolation. In order to detect the features present only in the ON spectra, we computed the ratio spectra $\mathrm{ON} / \mathrm{OFF}$ after the proper velocity correction; the SNR in the ratio spectra is lower than in individual frames, because the noise is summed up. It is also observed all along the $\mathrm{ON} / \mathrm{OFF}$ spectrum that the noise at the location of stellar lines is greater than in the local continuum by a factor of 3 to 10 (see Fig. 4); this is due to the imperfect interpolation of the flux, over the strong gradients of the divided line profiles.

\subsection{Instrumental effects}

To reach the requested quality, the data are extremely sensitive to any instrumental effect which would affect the 

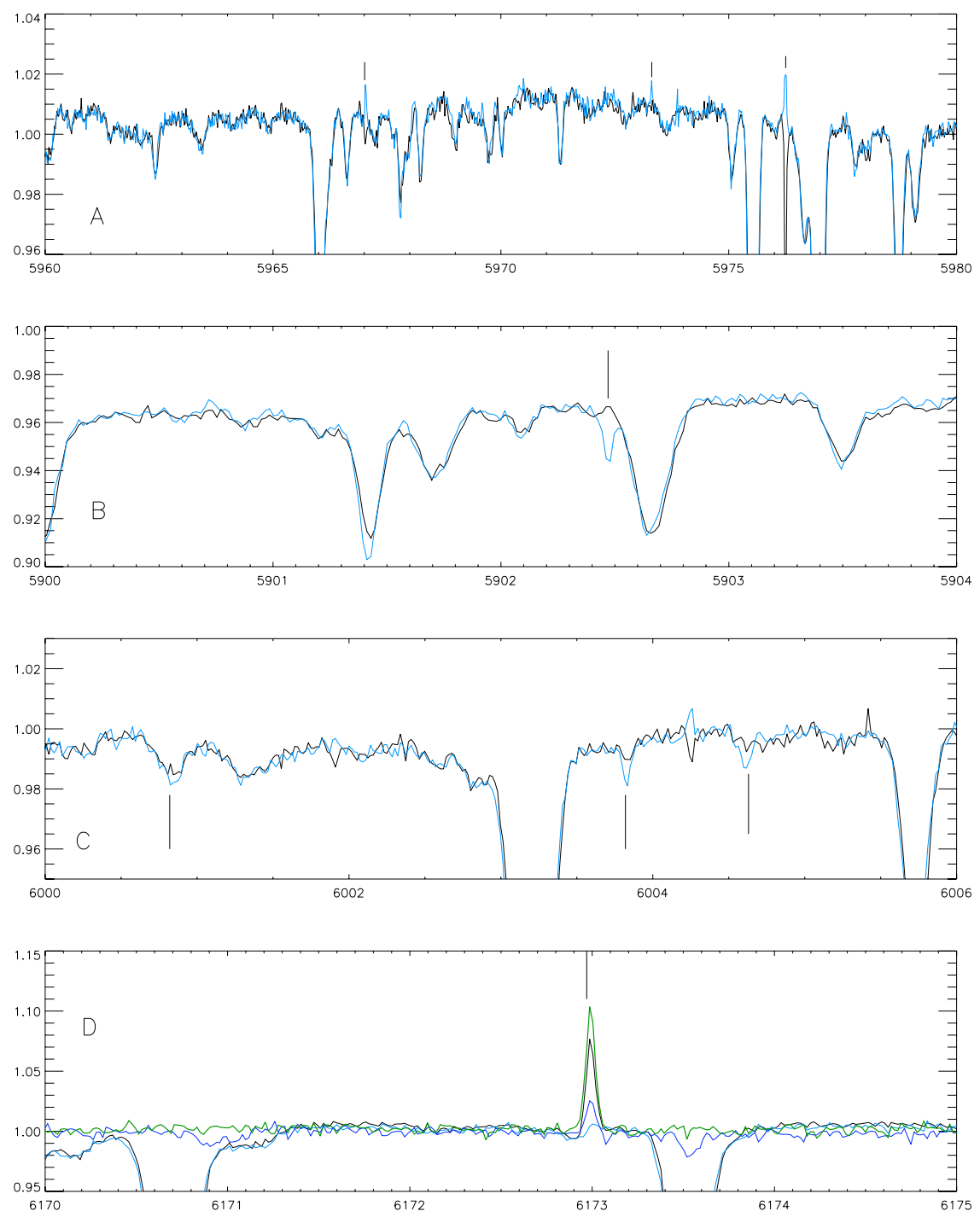

Fig. 3. Some pieces of normalized spectra ON (thick line), OFF (thin line) and reference star (dashed line). $X$ axis is in $\AA$. Several instrumental artefacts are shown: (A) The ON spectrum and the reference star's spectrum show a feature in absorption, whereas at the same location, the OFF spectrum shows an emission spike. This produces an artificial absorption line in the ON/OFF ratio spectrum; (B) The OFF spectrum shows an absorption feature whereas the reference spectrum shows an emission spike and the ON is pure continuum; (C) A false absorption feature is deeper in the OFF spectrum than in the ON and the reference spectra; (D) A strong spike is present in all spectra, but the ON spectrum. (E) An emission spike is visible in all spectra, with varying intensity

data taken on different dates. Figure 3 shows some examples of such defects, which were flagged as instrumental after inspection of the three individual exposures. The division of $\mathrm{ON}$ and $\mathrm{OFF}$ spectra revealed a lot of faint variations, sometimes appearing in absorption as would do an absorption feature due to the planet. For instance, a residual of a cosmic ray in the OFF spectrum will create an artificial absorption feature in the ratio (Fig. 3A). While cosmic rays are grossly removed by the optimal extraction method used by UVES pipeline, some residuals remain.

Most of the time, these few detected features could be attributed to CCD non-linear properties of pixel clusters ("black spots"), localized on the chip and recognizable on raw frames, before spectrum extraction (Fig. 3C). The two spectra taken during different nights and at variable seeing conditions, do not have exactly the same location on the CCD, and non-linear effects therefore do not affect the flux at same wavelengths. This produces a significant impact on the $\mathrm{ON} / \mathrm{OFF}$ ratio spectrum, when examined by eye.

\subsection{Rejection criteria}

Having discussed potential sources of artefacts, we want to stress the chosen criteria for considering a feature with a possible exospheric origin:

1. The feature should be seen in absorption in all three independent ON spectra, even with variable intensities (time variability is indeed expected); 

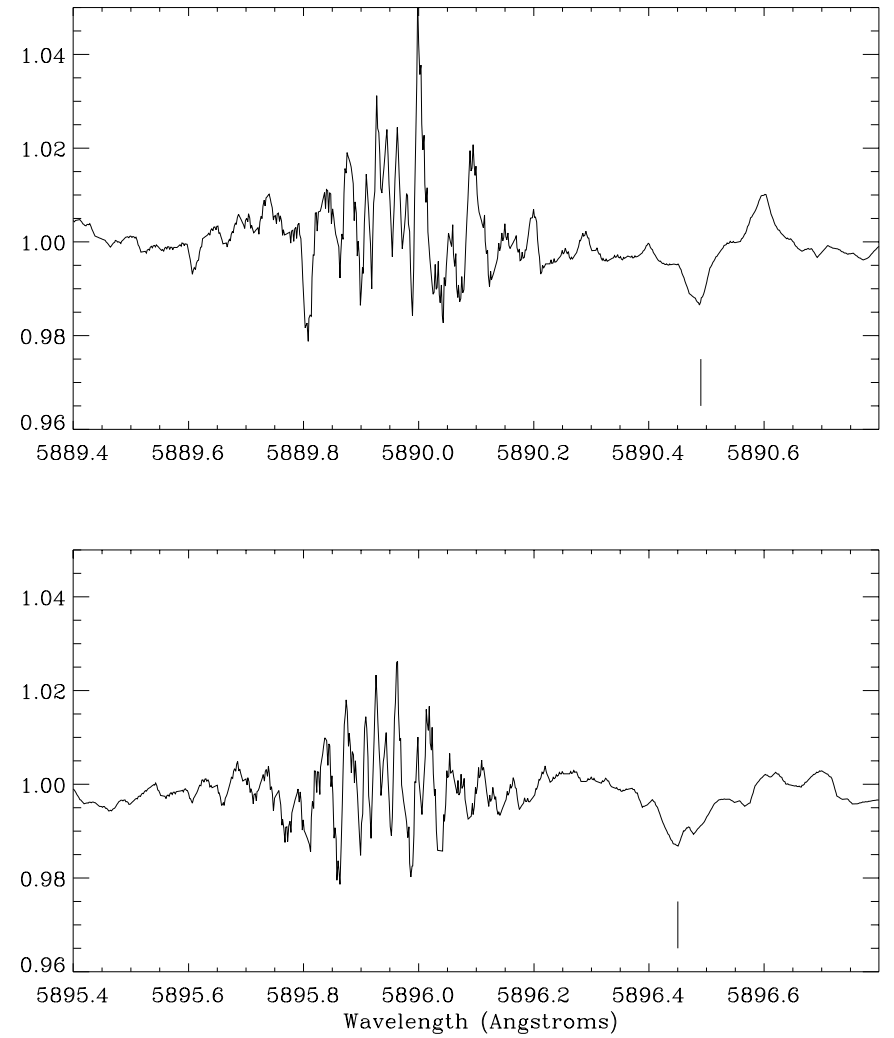

Fig. 4. The ON/OFF ratio spectrum around the NaI doublet region. The presence of stellar lines severely increases the local noise, making the detection of exospheric signatures difficult. The most prominent features at 5890.49 and $5896.45 \AA$ are separated by $6 \AA$ as the real NaI doublet, and should be inspected in further observations

2. The feature should be of much lower intensity (if torus like) or absent (if a tail or bridge of infalling matter) in all OFF spectra;

3. The integrated SNR of the flux over the feature should be larger than 3 . Weaker constraints can be considered, with some care, if the feature is visible on all on-transit exposures with slightly lower SNR;

4. The feature should be absent in the spectra of reference stars, providing these stars are actual calibration stars.

With such criteria, we confirm the rejection of the cases presented previously, which are clearly identified as instrumental and data-reduction artefacts.

In Fig. 5, an attempt to illustrate the actual SNR is shown. Two individual spectra have been divided (ON/OFF) after proper velocity corrections, and simulated absorption lines were injected in the ratio spectrum. This mimics a situation where the ON spectrum would show additional features due to the exospheric transit, in the spectrum range of highest SNR. The detection limit then depends on the assumed bandwidth and on a possible overlap with a stellar absorption line. A line of central depth of $1 \%$ and width $0.2 \AA$ would be detected with a SNR of 3 (see line A on the bottom plot). The ionic species we are searching for could be even narrower, which would make them more difficult to detect (line B on top plot).
As the line profile is unknown, we have to remain conservative and not allow a detection at a level less than $3 \sigma$.

\subsection{Discussion on a few signatures}

We have been looking for some expected absorption lines of HD 209458b's exosphere (Seager \& Sasselov 2000; Coustenis et al. 1997). A series of absorption lines from ionic species observed in comets have been searched for, without convincing detection. Only two cases are discussed here.

- The ON/OFF transmission spectrum shows an absorption line at $6146.81 \AA$, where we expect an $\mathrm{H}_{2} \mathrm{O}^{+}$feature. The line is variable in time and most prominent in the ON spectra. One of the reference stars (HD 224926) also exhibits a similar feature at the same location and with similar width, which makes its origin rather suspicious. The spectrum extraction is likely to produce varying artefacts, as seen in Sect. 4.2, e.g. if a dead zone of the CCD affects the star profile, whose extension across the detector may vary from one exposure to the other;

- The NaI doublet is expected to be strong in the transmission spectrum of HD 209458 (Seager \& Sasselov 2000). The relevant sections of the spectrum, shown in Fig. 4, are affected by stellar NaI absorption lines and by telluric lines. Vertical lines in Fig. 4 tentatively show the location of two possible absorptions in the ON spectrum. The physical separation of $6 \AA$ between both components of the doublet remarkably is observed between the pair of features at 5890.49 and $5896.45 \AA$. However, our criterion that the lines be of larger amplitude than the local noise is not fulfilled in this case and we cannot confirm that these features are due to the absorbing planetary exosphere. Moreover, these lines are not detected in the analysis of all individual $\mathrm{ON} / \mathrm{OFF}$ spectra.

\section{Discussion}

We analysed a preliminary data set of observations with high resolving power in the optical range of HD209458 during a planetary transit and off-transit phase. The following conclusions can be drawn:

- The achieved SNR is of the order of the detection limit of the expected features ( $1 \%$ deep) in the transmission spectrum. This limit, however, depends on the assumed line width, which is so far poorly constrained. A $0.2 \AA$ wide line would be detected in the red at a $\mathrm{SNR}$ of 3 if its central depth is $1 \%$. At shorter wavelengths, the achieved SNR is lower and does not allow the detection of 2 to $3 \%$ depth features;

- We can derive a rough upper limit of the exosphere's scale height $H$ from the achieved SNR of our data set. From simple geometrical considerations, we derive that optically thin absorption lines have a normalized flux 

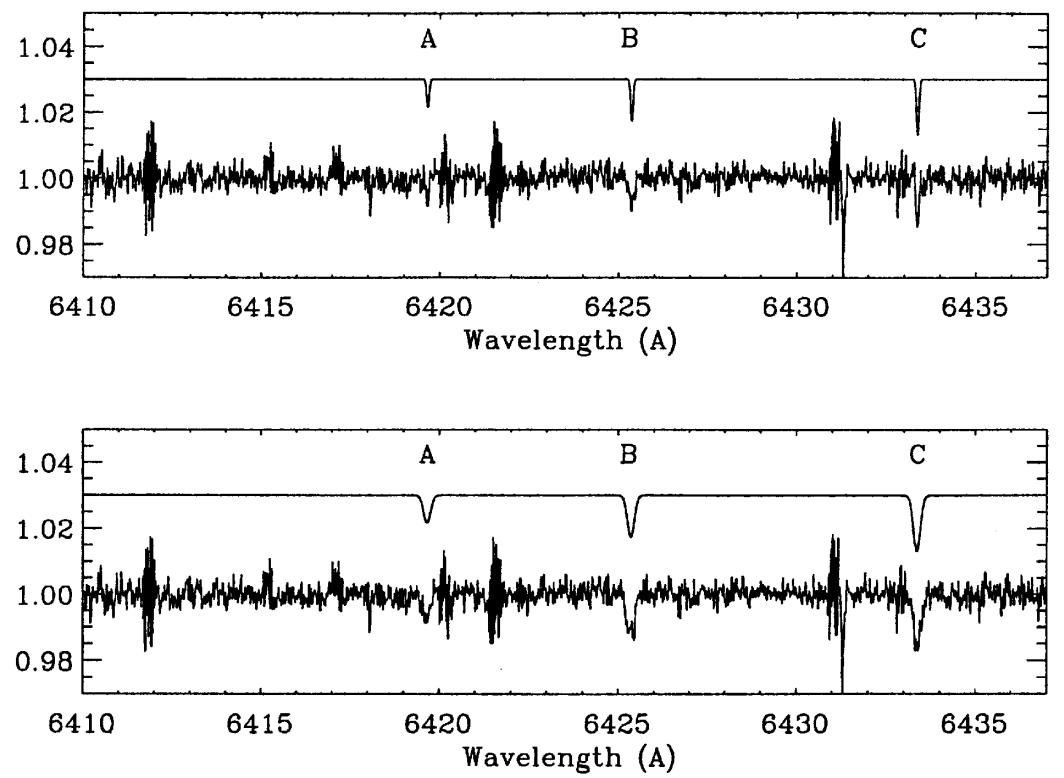

Fig. 5. This figure illustrates the detection limits achieved in our highest quality spectrum ("RU"), and its dependence on the line width. In an ON/OFF ratio spectrum of individual exposures, simulated absorption lines have been injected. The simulated gaussian lines are shown, with a +0.03 shift in $\mathrm{Y}$ for clarity. Three lines of varying central depth are shown. The detection limit is illustrated by the B line (central depth $1.3 \%$, bandwith $0.1 \AA$ ) on the top spectrum and the A line (central depth $0.8 \%$, bandwith $0.22 \AA$ ) on the bottom spectrum, both detected at $3 \sigma$

$\Delta F / F$ of the order of $\sqrt{H R_{\mathrm{pl}}} R_{\mathrm{pl}} / R_{*}^{2}$. The maximum theoretical SNR, 500 in the redder part of the UVES spectrum, thus leads to a maximum scale height of $\simeq 1500 \mathrm{~km}$. This estimate is relevant for a bound spherical exosphere. The case of a giant cometary-like tail cannot be easily constrained by our lack of detection;

- If the exosphere remains undetected even in higherquality data sets, it may be due to: (1) a weak stellar wind which does not perturb the azimuthal symmetry of the accretion ring; (2) a tenuous planet exosphere; (3) an homogeneous torus of matter analogous to the Jupiter/Io case;

- The difficult search for an exospheric signature of HD 209458b in the optical, superimposed on a rich stellar spectrum, requires a more extended time coverage and higher SNR data set. We have obtained more telescope time on VLT+UVES for this project and the observations are currently under reduction and analysis (Coustenis et al., in preparation). The experience gained in previous similar observations and with this preliminary follow-up of HD 209458b's transit would be a very valuable support for further searches. A positive detection of the exosphere of an extra-solar planet would represent a breakthrough in the knowledge of this object's composition;

- Other attempts to detect the exosphere of HD 209458b have been conducted (Bundy \& Marcy 2000; Rauer et al. 2000b). Both experiments were also preliminary, i.e. they did not cover the complete transit and were taken at relatively high airmass. No exosphere has been detected so far. Our data set is of higher signal-tonoise ratio and thus a stronger constraint on the exosphere's detectability is derived;
- A complex chemistry is to be expected in the planetary environment, due to high temperature and pressure variations between day and night sides of the planet (Guillot 2000), and from interactions with the closeby star. Modelling the composition of the exosphere, including photo-chemistry, is a crucial tool for a refined search for specific features. It may be a future key to enhance the detection, e.g. by cross-correlation techniques, of a faint signature originating in a chemicallyrich exosphere.

Acknowledgements. We are grateful to Sandro D'Odorico and the UVES Commissioning team to have allowed and executed this observation. Many thanks to the referee for useful comments.

\section{References}

Brace, L., Kasprzak, W., Taylor, H., et al. 1987, JGR, 92, 15 Bundy, K., \& Marcy, G. 2000, PASP, preprint

Brown, et al. 2001, submitted to ApJ

Charbonneau, D., Brown, T., Mayor, M., \& Mazeh, T. 1999 November 22, IAU circular 7315

Charbonneau, D., Brown, T., Latham, D., \& Mayor, M. 2000a, ApJ, 529, L45

Chassefière, E. 1996, J. Geophys. Res., 101, 26039

Chassefière, E. 1997, Icarus, 229, 232

Coustenis, A., Schneider, J., Bockelée-Morvan, D., et al. 1997, in Planets beyond the Solar System and the Next Generation of Space Missions, ed. D. Soderblom, ASP, Conf. Ser., 119, 101

Coustenis, A., Schneider, J., Wittemberg, R., et al. 1998, in Brown dwarfs and Extrasolar planets, ASP conf. Ser., 134, ed. Rebolo, Martín, \& Zapatero Osorio, 296 
Coustenis, A., et al. 2000, in EGS meeting, Nice, France

Delbouille, L., Rolan, G., \& Neven, L. 1973, Atlas photométrique du spectre solaire 3000-10000Ang., Liège

D'Odorico, S., Cristiani, S., Dekker, H., et al. 2000, SPIE Proceedings 4005, J. Bergeron edt, in press

Goukenleuque, C., Bézard, B., Joguet, B., \& Lellouch, E. 2000, Icarus, 143, 308

Guillot, T., Burrows, A., Hubbard, W., et al. 1996, ApJ, 459, L35

Guillot, T., et al. 2000, in proceedings of the "IAU Symposium S202", Manchester 2000

Hatzes, A., Kürster, M., Cochran, W., Dennerl, K., \& Döbereiner, S. 1996, JGR, 101, 9285

Henry, G., Marcy, G., Butler, R., \& Vogt, S. 2000, ApJ, 529, L41
Lin, D. N. C., Bodenheimer, P., \& Richardson, D. C. 1996, Nature, 380, 606

Marcy, G., \& Butler, P. 1996, ApJ, 464, L147

Mazeh, T., Naef, D., Torres, G., et al. 2000, ApJ, 532, L55

Mayor, M., \& Queloz, D. 1995, Nature, 378, 355

Queloz, D., Eggenberger, A., Mayor, M., et al. 2000, A\&A, in press

Rauer, H., Bockelée-Morvan, D., Coustenis, A., et al. 2000a, A\&A, 355, 573

Rauer, H. et al. 2000b, in proceedings of the "IAU Symposium S202", Manchester 2000

Schneider, J., Rauer, H., et al. 1998, in Brown Dwarfs and Extrasolar Planets, ed. Rebolo et al., ASP Conf. Ser., 134, 241

Seager, S., \& Sasselov, D. 2000, ApJ, 537, 916 